\title{
Quantum Interference Controls the Electron Spin Dynamics in $\boldsymbol{n}$-GaAs
}

\author{
V. V. Belykh, ${ }^{1,2, *}$ A. Yu. Kuntsevich, ${ }^{2,3}$ M. M. Glazov, ${ }^{4,5}$ K. V. Kavokin, ${ }^{4,5}$ D. R. Yakovlev, ${ }^{1,4}$ and M. Bayer ${ }^{1,4}$ \\ ${ }^{1}$ Experimentelle Physik 2, Technische Universität Dortmund, D-44221 Dortmund, Germany \\ ${ }^{2}$ P.N. Lebedev Physical Institute of the Russian Academy of Sciences, 119991 Moscow, Russia \\ ${ }^{3}$ National Research University Higher School of Economics, 101000 Moscow, Russia \\ ${ }^{4}$ Ioffe Institute, Russian Academy of Sciences, 194021 St. Petersburg, Russia \\ ${ }^{5}$ Spin Optics Laboratory, St. Petersburg State University, 199034 St. Petersburg, Russia
}

(Received 15 March 2018; revised manuscript received 18 May 2018; published 23 July 2018)

\begin{abstract}
Manifestations of quantum interference effects in macroscopic objects are rare. Weak localization is one of the few examples of such effects showing up in the electron transport through solid state. Here, we show that weak localization becomes prominent also in optical spectroscopy via detection of the electron spin dynamics. In particular, we find that weak localization controls the free electron spin relaxation in semiconductors at low temperatures and weak magnetic fields by slowing it down by almost a factor of two in $n$-doped GaAs in the metallic phase. The weak localization effect on the spin relaxation is suppressed by moderate magnetic fields of approximately $1 \mathrm{~T}$, which destroy the interference of electron trajectories, and by increasing the temperature. The weak localization suppression causes an anomalous decrease of the longitudinal electron spin relaxation time $T_{1}$ with magnetic field, in stark contrast with the well-known magnetic-field-induced increase in $T_{1}$. This is consistent with transport measurements, which show the same variation of resistivity with magnetic field. Our discovery opens up a vast playground to explore quantum magnetotransport effects optically in the spin dynamics.
\end{abstract}

DOI: 10.1103/PhysRevX.8.031021

\section{INTRODUCTION}

The design of future spintronic and optospintronic devices requires a detailed understanding of the correlation between the electron conductivity and spin relaxation in prospective material systems, such as semiconductors. The electron spin relaxation in semiconductors depends strongly on whether electrons are itinerant or localized [1,2]. Across the metal-toinsulator transition (MIT), the spin relaxation changes as dramatically as the conductivity [2,3]. Indeed, in the insulating phase, both conductivity and spin relaxation critically depend on the overlap of the wave functions of donor-bound electrons at low temperatures and on the number of delocalized electrons at higher temperatures. In the metallic phase, in semiconductors without an inversion center, with GaAs as a prototype system, the spin relaxation is governed by spin-orbit coupling (Dyakonov-Perel mechanism) [4] and, similarly to the conductivity, the spin relaxation becomes suppressed by electron scattering events. The spin

\footnotetext{
*vasilii.belykh@tu-dortmund.de

†glazov@coherent.ioffe.ru
}

Published by the American Physical Society under the terms of the Creative Commons Attribution 4.0 International license. Further distribution of this work must maintain attribution to the author(s) and the published article's title, journal citation, and DOI.
Subject Areas: Condensed Matter Physics, Quantum Physics, Spintronics relaxation rate is closely related to the electron diffusion coefficient [5-7], so that charge transport phenomena are generally expected to manifest also in spin relaxation processes [8-11]. The situation becomes particularly involved in the vicinity of the MIT, where quantum effects become important $[12,13]$.

While the mechanisms of electron spin relaxation in semiconductors were largely clarified in theory back in the 1970s [14], for a long time, experiments could access the electron spin dynamics only via the Hanle effect near zero magnetic field. Since the 1990s, more advanced techniques have become available, such as pump-probe methods analyzing the Kerr (or Faraday) rotation $[15,16]$ or polarization-resolved photoluminescence [17-20], and elaborated methods like resonant spin amplification [21,22], spin noise spectroscopy [23-25], and spin inertia reorientation [26]. Each of these tools has limitations related to the achievable time resolution, the addressable time range, or the applicable magnetic field. So far, access to the relation between the electron diffusion and spin relaxation in the vicinity of the MIT was hindered by experimental limitations. Only recently was the pump-probe technique extended to facilitate direct measurements of arbitrarily long spin dynamics with picosecond time resolution across a wide range of magnetic fields [27]. 
On the other hand, the transport properties of semiconductors that directly provide information about electron diffusion are rather easily accessible in experiment. In weak magnetic fields, the low temperature magnetoresistance is negative due to the weak localization effect: The magnetic field destroys the phase coherence of interfering paths and increases the electron diffusion coefficient [28-35]. The spinorbit interaction has a pronounced impact on the low-field magnetoresistance leading to positive magnetoresistance, i.e., antilocalization, if the spin coherence of electrons is lost faster than their phase [28]. Although weak localization or antilocalization is expected to emerge in the spin dynamics $[13,36]$, it has not been identified in experiments so far.

In this paper, we demonstrate that weak localization significantly slows down the itinerant electron spin relaxation in the Dyakonov-Perel mechanism. Using the extended pump-probe Faraday rotation technique, we study the longitudinal electron spin relaxation time $T_{1}$ as a function of external magnetic field in $n$-doped metallic bulk GaAs. While the classical theory [37] predicts an increase of $T_{1}$ with increasing field, mainly due to the cyclotron motion of the free carriers, we observe an anomalous decrease of $T_{1}$ in moderate fields $B \lesssim 1 \mathrm{~T}$. From transport measurements done on the same samples, we observe that the negative magnetoresistance is correlated with the anomalous magnetic field dependence of $T_{1}$. We develop a theoretical model of the weak localization effect in the spin relaxation of bulk semiconductors and find very good agreement between the calculations and experimental data. Our results establish a strict relation between the electron diffusion and spin relaxation in metallic systems in the vicinity of the MIT. Thereby all-optical access to weak localization is provided and a tool to probe locally electron transport phenomena is developed.

\section{EXPERIMENTAL DETAILS}

The results are obtained on Si-doped GaAs samples with electron concentrations of $n_{\mathrm{e}}=5.5 \times 10^{14} \mathrm{~cm}^{-3}(2-\mu$ m-thick layer grown by the molecular-beam epitaxy), $3.7 \times 10^{16} \mathrm{~cm}^{-3}$, and $7.1 \times 10^{16} \mathrm{~cm}^{-3}$ (140- and $170-\mu \mathrm{m}$-thick bulk wafers, respectively).

For optical measurements, the samples are placed in the variable temperature insert of a split-coil magnetocryostat $(T=2-25 \mathrm{~K})$. Magnetic fields up to $6 \mathrm{~T}$ are applied parallel to the light propagation direction that is parallel to the sample growth axis (Faraday geometry). The extended pump-probe Kerr (Faraday) rotation technique is used to study the electron spin dynamics. It is a modification of the standard pump-probe Kerr (Faraday) rotation technique, where circularly polarized pump pulses generate carrier spin polarization, which is then probed by the Kerr (Faraday) rotation of linearly polarized probe pulses after reflection (transmission) from (through) the sample. Implementation of pulse picking for both pump and probe beams in combination with a mechanical delay line allows us to scan microsecond time ranges with picosecond time resolution. Details of the technique are given in Ref. [27].

Here, a Ti:Sapphire laser emits a train of 2-ps pulses with a repetition rate of $76 \mathrm{MHz}$ (repetition period $T_{\mathrm{R}}=13.1 \mathrm{~ns}$ ). The pump protocol uses a single pulse per excitation period. The separation between these pulses is $80 T_{R}, 160 T_{R}$, or $320 T_{\mathrm{R}}$ in order to clearly exceed the characteristic time of spin polarization decay. The sample with donor concentration $n_{\mathrm{e}}$ of $5.5 \times 10^{14} \mathrm{~cm}^{-3}$ is studied in reflection geometry (Kerr rotation) with the laser wavelength set to $819 \mathrm{~nm}$, close to the donor-bound exciton resonance. The samples with $n_{\mathrm{e}}=3.7 \times 10^{16} \mathrm{~cm}^{-3}$ and $7.1 \times 10^{16} \mathrm{~cm}^{-3}$ are studied in transmission geometry (Faraday rotation), with the laser wavelength set to $829 \mathrm{~nm}$.

Magnetoresistance measurements were performed using a standard four-terminal technique with a lock-in amplifier. The measurement current $(36 \mathrm{~Hz}, 100 \mu \mathrm{A})$ was checked not to overheat the sample at the lowest temperature. Ohmic contacts (with an almost $T$-independent resistance of about $100 \mathrm{ohms}$ ) were obtained by annealing of indium drops on top of the preliminary scratched wafer $\left(10 \mathrm{~min}\right.$ at $400^{\circ} \mathrm{C}$ in vacuum). A PPMS-9 cryostat and Cryogenics CFMS-16 system were used to set the temperature $(2-40 \mathrm{~K})$ and magnetic field (up to $6 \mathrm{~T}$ ). The magnetic field perpendicular to the sample surface and current direction was swept from positive to negative values with subsequent symmetrization of the data to compensate for inevitable contact misalignment.

\section{RESULTS AND DISCUSSION}

\section{A. Experiment}

The circularly polarized pump laser pulse creates spin polarization along the magnetic field (Faraday geometry $\boldsymbol{B}\|\boldsymbol{z}\|[001])$, which can be detected by the delayed probe laser pulse via Faraday rotation of its linear polarization. Figure 1(a) shows the dynamics of the spin polarization for exemplary values of the external magnetic field $B=0,2$, and $6 \mathrm{~T}$ for the metallic sample with electron concentration $n_{\mathrm{e}}=3.7 \times 10^{16} \mathrm{~cm}^{-3}$, which is somewhat above the MIT threshold, $n_{\mathrm{e}}^{\mathrm{MIT}} \approx(1-2) \times 10^{16} \mathrm{~cm}^{-3}$. The signal decays monoexponentially with the longitudinal spin relaxation time $T_{1}$. It is seen from Fig. 1(a) that, as the magnetic field grows, $T_{1}$ first decreases, reaches a minimum, and then increases. The nonmonotonic dependence of $T_{1}(B)$ with a minimum at about $1.5 \mathrm{~T}$ is further substantiated in Fig. 1(b) by the solid spheres. The minimum in the $T_{1}(B)$ dependence becomes less pronounced for the sample with even higher carrier concentration, while for the samples with lower donor concentrations, below MIT, $T_{1}$ monotonically increases with increasing $B$ [the open circles in Fig. 1(b)].

To investigate the anomalous $T_{1}(B)$ dependence further, we perform measurements at different temperatures with the results summarized in Fig. 2(a). Interestingly, the minimum in the $T_{1}(B)$ dependence at increased $B$ (or, alternatively, peak at $B=0$ ) is observed only at low temperatures 

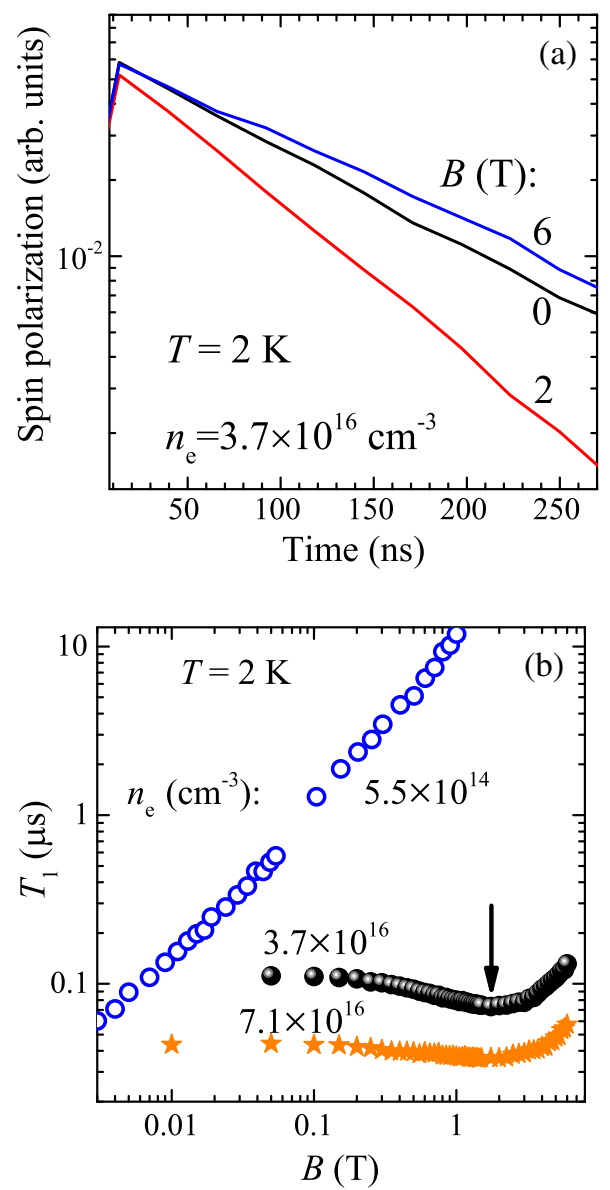

FIG. 1. Longitudinal spin relaxation. (a) Dynamics of the electron spin polarization (measured as Faraday rotation signal) at different magnetic fields for the $n$-GaAs sample with $n_{\mathrm{e}}=3.7 \times 10^{16} \mathrm{~cm}^{-3}$. (b) Magnetic field dependence of the longitudinal relaxation time $T_{1}$ for samples with different donor concentrations. The arrow indicates the minimum in the $T_{1}(B)$ dependence. (a),(b) Temperature $T=2 \mathrm{~K}$.

$T \lesssim 14 \mathrm{~K}$. Furthermore, with increasing temperature, the minimum becomes less pronounced due to the decrease of the zero-field $T_{1}$ value. The decrease of $T_{1}$ with magnetic field or temperature increase is unexpected in view of existing theories of free-electron spin relaxation in semiconductors $[4,7,14,37]$. This calls for a detailed modeling of the spin relaxation process, which is presented below.

\section{B. Model}

In GaAs-like semiconductors, being in the metallic phase, the spin relaxation is controlled by the Dyakonov-Perel mechanism [4,14]: The electron spin precesses around the effective, spin-orbit coupling-induced magnetic field, and the spin precession is randomized by scattering events. The spin dynamics is described in the framework of a kinetic equation for the spin distribution function $\boldsymbol{s}_{k}$ [4,37-39]:

$$
\frac{\partial \boldsymbol{s}_{k}}{\partial t}+\Lambda_{k}\left\{\boldsymbol{s}_{k}\right\}+\boldsymbol{s}_{\boldsymbol{k}} \times \boldsymbol{\Omega}_{k}=Q\left\{\boldsymbol{s}_{k}\right\}
$$
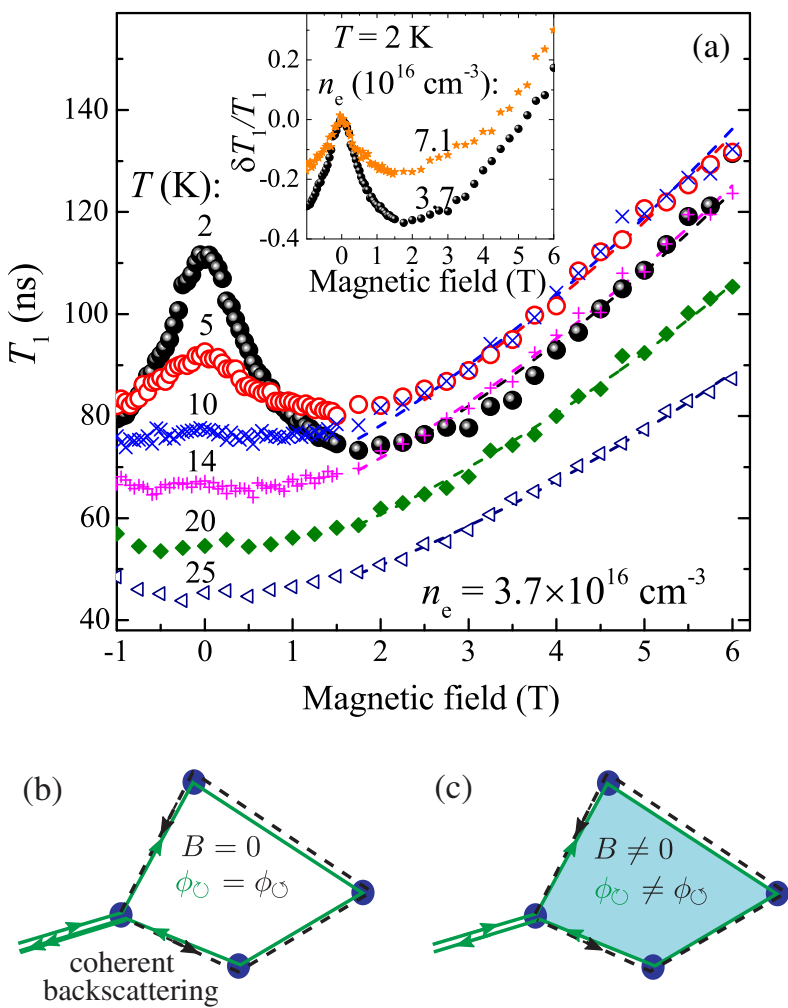

FIG. 2. Effect of weak localization on longitudinal spin relaxation time $T_{1}$. (a) Magnetic field dependence of $T_{1}$ at different temperatures. $n_{\mathrm{e}}=3.7 \times 10^{16} \mathrm{~cm}^{-3}$. The dashed lines show fits to the experimental data with Eq. (5). Inset shows relative variation of $T_{1}$ with magnetic field for metallic samples with different electron concentrations. (b) Scheme of constructive interference of clockwise and counterclockwise electron paths, starting at the same impurity and related by the time reversal symmetry. The interference gives rise to the weak localization effect by increasing the backscattering efficiency. (c) The interference between the same paths as in Fig. 2(b) is destroyed by the magnetic field due to the extra phase acquired by the electron traveling clockwise and counterclockwise.

Each term in Eq. (1) has a transparent physical meaning. The operator $\Lambda_{\boldsymbol{k}}=-\boldsymbol{\omega}_{c}[\boldsymbol{k} \times \partial / \partial \boldsymbol{k}]$ describes the electron cyclotron motion in the external magnetic field, where $\boldsymbol{\omega}_{c}=$ $e \boldsymbol{B} / m c$ is the cyclotron frequency, $m$ is the electron effective mass, and $e$ is the electron charge (the Zeeman splitting is neglected). The term $s_{k} \times \boldsymbol{\Omega}_{k}$ describes the precession of the electron spin around the effective magnetic field arising due to the spin-orbit interaction in a system with bulk inversion asymmetry. The corresponding precession frequency $\boldsymbol{\Omega}_{\boldsymbol{k}}$ is cubic on the electron wave vector $\boldsymbol{k}$. In the last term in Eq. (1), $Q\left\{\boldsymbol{s}_{\boldsymbol{k}}\right\}$ is the collision integral, i.e., the operator describing the redistribution of electrons between different states in $\boldsymbol{k}$-space. It takes into account the electron scattering and can be generally presented as

$$
Q\left\{\boldsymbol{s}_{\boldsymbol{k}}\right\}=\sum_{\boldsymbol{k}^{\prime}}\left(W_{\boldsymbol{k} \boldsymbol{k}^{\prime}} \boldsymbol{s}_{\boldsymbol{k}^{\prime}}-W_{\boldsymbol{k}^{\prime} \boldsymbol{k}} \boldsymbol{s}_{\boldsymbol{k}}\right),
$$


describing the balance between the processes where an electron leaves the state with wave vector $\boldsymbol{k}^{\prime}$ and is promoted to the state with wave vector $\boldsymbol{k}$ with the rate $W_{\boldsymbol{k} \boldsymbol{k}^{\prime}}$ and vice versa, accordingly. For the elastic scattering by the central potential of ionized donors relevant for the studied system, $W_{\boldsymbol{k} \boldsymbol{k}^{\prime}}=W_{\boldsymbol{k}^{\prime} \boldsymbol{k}}$, and relaxation of different angular harmonics $Y_{l m}\left(\vartheta_{k}, \varphi_{k}\right)$ of the distribution function $(\vartheta$ and $\varphi$ are the angles of the wave vector) occurs independently [4]. Thus, for the spin distribution $s_{k}=\delta s_{k} Y_{l m}\left(\vartheta_{k}, \varphi_{k}\right)$, the collision integral $Q\left\{\delta \boldsymbol{s}_{k} Y_{l m}\left(\vartheta_{\boldsymbol{k}}, \varphi_{\boldsymbol{k}}\right)\right\}=-\tau_{l}^{-1} \delta \boldsymbol{s}_{k} Y_{l m}\left(\vartheta_{\boldsymbol{k}}, \varphi_{k}\right)$, and it is described by a set of relaxation times $\tau_{l}(l=1,2,3, \ldots)$ :

$$
\frac{1}{\tau_{l}}=\sum_{\boldsymbol{k}^{\prime}} W_{\boldsymbol{k}^{\prime} \boldsymbol{k}}\left[1-P_{l}\left(\cos \vartheta_{\boldsymbol{k}^{\prime}}\right)\right],
$$

responsible for the relaxation of different angular harmonics of the distribution function; $P_{l}(x)$ is the corresponding Legendre polynomial. Note that $\tau_{1}=\tau_{p}$ describes the momentum relaxation of electrons. In a classical approach, these relaxation times are independent of the magnetic field.

The electron scattering slows down the spin relaxation due to randomization of the spin precession around the spin-orbit magnetic field: Between the scattering acts, the electron spin rotates by a small angle $\sim \Omega_{k} \tau$ ( $\tau$ is the characteristic relaxation time), while the scattering processes change the wave vector $\boldsymbol{k}$ and, correspondingly, the spin precession frequency $\boldsymbol{\Omega}_{\boldsymbol{k}}$, reducing the cumulative spin rotation angle. It follows from the solution of Eq. (1) that the longitudinal spin relaxation time for degenerate electrons in bulk GaAs at $B=0$ takes the form [4]

$$
T_{1}(0)=\frac{105}{32 \alpha^{2}} \frac{\hbar^{2} E_{g}}{E_{F}^{3} \tau_{3}},
$$

where $E_{F}$ is the electron Fermi energy, $E_{g}=1.52 \mathrm{eV}$ is the band gap energy, $\alpha \approx 0.063$ is the dimensionless Dresselhaus constant for GaAs, recalculated from data in Refs. [40,41], and $\tau_{3}$ is the relaxation time of third angular harmonics of the electron distribution over momentum given by Eq. (3).

A similar suppression of the spin relaxation takes place due to the cyclotron motion of the electron in an external magnetic field accounted for by the operator $\Lambda_{k}\left\{s_{k}\right\}$ in Eq. (1). Indeed, the field induces a rotation of the electron velocity and the wave vector $\boldsymbol{k}$, thus, resulting in a rotation of the effective magnetic field $\propto \boldsymbol{\Omega}_{\boldsymbol{k}}$. In this way, the magnetic field acts as an extra scattering source and slows down the spin relaxation $[37,42]$. The magnetic field dependence of $T_{1}$ was calculated in Ref. [37]:

$$
\frac{T_{1}(B)}{T_{1}(0)}=\frac{\left[1+\left(\omega_{c} \tau_{3}\right)^{2}\right]\left[1+9\left(\omega_{c} \tau_{3}\right)^{2}\right]}{1+6\left(\omega_{c} \tau_{3}\right)^{2}} \approx 1+4 \omega_{c}^{2} \tau_{3}^{2} .
$$

The last approximate equality in Eq. (5) holds for $\omega_{c} \tau_{3} \ll 1$. Equation (5) clearly demonstrates an increase in the spin relaxation time $T_{1}$ with growing magnetic field. This expression with the temperature-independent $\tau_{3} \approx 40 \mathrm{fs}$ describes the experimental data at $B \gtrsim 2 \mathrm{~T}$ [the dashed lines in Fig. 2(a)]. From the value of $T_{1}$ extrapolated to $B=0$, we obtain after Eq. (4) almost the same $\tau_{3}$ as the value obtained above from the $B$-dependence.

The classical theory of the Dyakonov-Perel spin relaxation mechanism, expressed by Eqs. (4) and (5), as well as additional possible mechanisms of spin relaxation due to the $g$-factor spread [43], cannot, however, explain the sizable decrease of the spin relaxation time $T_{1}$ in rather weak magnetic fields $B \lesssim 1 \mathrm{~T}$ and at low temperatures $T \lesssim 14 \mathrm{~K}$. Clearly, other effects, not accounted for by the approach in Refs. [4,7,14,37-39], must play an important role in our experiment. In fact, in the derivation of Eqs. (4) and (5), the electron dynamics is assumed to be classical, i.e., the inequality $E_{F} \tau_{p} / \hbar \gg 1$ is assumed to hold. For relatively low electron densities, $E_{F} \tau_{p} / \hbar$ just slightly exceeds unity and quantum effects start to play a role. In particular, for an electron traveling through a disordered medium, the interference between classical trajectories, as schematically depicted in Fig. 2(b), becomes important. For electron waves traveling clockwise and counterclockwise through the same configuration of impurities, the phases acquired on these two paths, $\phi_{\circlearrowright}=\phi_{\circlearrowleft}=\oint \boldsymbol{k} d \boldsymbol{l}$, are the same. As a result, the two paths shown by the solid and dashed lines interfere constructively, leading to coherent backscattering. In effect, the scattering efficiency by the impurities increases ( $\tau_{p}$ decreases) and the electron propagation slows down. This is the weak localization effect, signifying the onset of the MIT with decreasing electron density. Importantly, a magnetic field destroys the constructive interference owing to the extra phase proportional to the field flux through the trajectory acquired by the diffusing electron; see Fig. 2(c). Indeed, for clockwise and counterclockwise propagation, the field-induced phases are opposite; hence, the magnetic field suppresses the weak localization [28,33-35,44].

In order to account for the interference effect, we follow the semiclassical approach where, as illustrated in Figs. 2(b) and 2(c), the quantum effects are accounted for by renormalization of the cross section for electron scattering by the impurity $[13,36,45]$. The momentum relaxation time $\tau_{p}$ acquires a correction $\delta \tau_{p}$ of the form

$$
\frac{\delta \tau_{p}}{\tau_{p}}=-\frac{2 m D}{\pi \hbar n_{\mathrm{e}}} \sum_{s, s^{\prime}= \pm 1 / 2} \mathcal{C}_{s s^{\prime} s^{\prime} s}(\boldsymbol{r}=0),
$$

where $D=v_{F}^{2} \tau_{p} / 3$ is the electron diffusion coefficient, and $v_{F}$ is the Fermi velocity. In Eq. (6), $\mathcal{C}_{s_{1} s_{2} s_{3} s_{4}}(\boldsymbol{r}=0)$ is the Cooperon matrix describing the electron interference along the closed loops, which is calculated via a standard diagram technique [28]. Similarly, the interference effects modify the relaxation time of the third angular harmonics of the spin distribution function $\tau_{3}$, which defines the spin relaxation time [Eq. (4)], as 


$$
\frac{\delta \tau_{3}}{\tau_{3}}=-\frac{2 m D}{\pi \hbar n_{\mathrm{e}}} \sum_{s, s^{\prime}= \pm 1 / 2} \mathcal{C}_{s s^{\prime} s^{\prime} s}(\boldsymbol{r}=0)\left(2 \delta_{s s^{\prime}}-1\right) .
$$

The factor $\left(2 \delta_{s s^{\prime}}-1\right)$ is due to the spin vortices in the corresponding diagrams $[13,36]$.

The Cooperon matrix, i.e., the sum of maximally crossed diagrams, describes the spin-dependent probability $P_{\text {ret }}$ of an electron to return to the initial point after an arbitrary number of collisions conserving its phase, which is the probability to pass through a loop in the real space [Fig. 2(b)]. Qualitatively, the interference of electron waves propagating clockwise and counterclockwise on the loops [Fig. 2(b)] gives rise to the coherent backscattering effect and modifies the rate of the scattering by an impurity $W_{\boldsymbol{k} k^{\prime}}$. It gives rise to a sharp peak in $W_{\boldsymbol{k} \boldsymbol{k}^{\prime}}$ at $\boldsymbol{k}^{\prime} \approx-\boldsymbol{k}$, i.e., for backscattering [45]. The interference-induced contribution $\delta W_{\boldsymbol{k} \boldsymbol{k}^{\prime}}=W_{\boldsymbol{k} \boldsymbol{k}^{\prime}}-W_{\boldsymbol{k} \boldsymbol{k}^{\prime}}^{c l} \propto P_{\text {ret }}$, where $W_{k k^{\prime}}^{c l}$ is the classical value found without interference effects, is proportional to the return probability. It gives rise to the corrections $\delta \tau_{l}$ to the relaxation times $\tau_{l}$ in Eq. (3). Both $\delta W_{\boldsymbol{k} k^{\prime}}$ and $\delta \tau_{l}$ are determined by the interference of the trajectories in Fig. 2(b). The magnetic field destroys the interference and suppresses $\delta \tau_{l}$, affecting the electron transport and spin dynamics.

We introduce the phase relaxation time $\tau_{\phi}$ associated with inelastic electron-electron or electron-phonon scattering processes, and we consider hereafter the diffusive regime where $\tau_{\phi} \gg \tau_{p}, \tau_{3}$ and the magnetic length $l_{B}=$ $\sqrt{\hbar c /(|e| B)}$ exceeds by far the mean free path, $l_{B} \gg v_{F} \tau_{p}$. Moreover, we impose the condition of rather weak spinorbit interaction, $T_{1}(0) \gg \tau_{\phi}$, meaning that the electron spin is conserved during passage through the closed loops in which the interference takes place. As a result, we have

$$
\frac{\delta T_{1}(B)}{T_{1}(0)}=\frac{\delta \rho(B)}{\rho(0)}=-\frac{m}{2 \pi^{2} \hbar n_{\mathrm{e}} \tau_{p}} \sqrt{\frac{|e| B}{\hbar c}} F_{3}\left(\frac{B_{\phi}}{4 B}\right) .
$$

Here, $B_{\phi}=\hbar c /\left(|e| l_{\phi}^{2}\right), l_{\phi}=\sqrt{D \tau_{\phi}}$ is the phase relaxation length, and the function $F_{3}(x)$ is defined as [33-35]

$$
F_{3}(x)=\sum_{n=0}^{\infty}\left[2(\sqrt{n+1+x}-\sqrt{n+x})-\frac{1}{\sqrt{n+1 / 2+x}}\right] .
$$

Note that, for $x \ll 1, F_{3}(x) \approx 0.605$ and, for $x \gg 1$, $F_{3}(x) \approx 1 /\left(48 x^{3 / 2}\right)$.

\section{Discussion and comparison of electron spin dynamics and transport}

To independently experimentally confirm the presence of weak localization and estimate its magnitude in the considered system, we have also measured the magnetoresistance on the same samples [see Fig. 3(a)]. The low-field negative magnetoresistance is clearly seen; in agreement with previous works, it arises from the weak
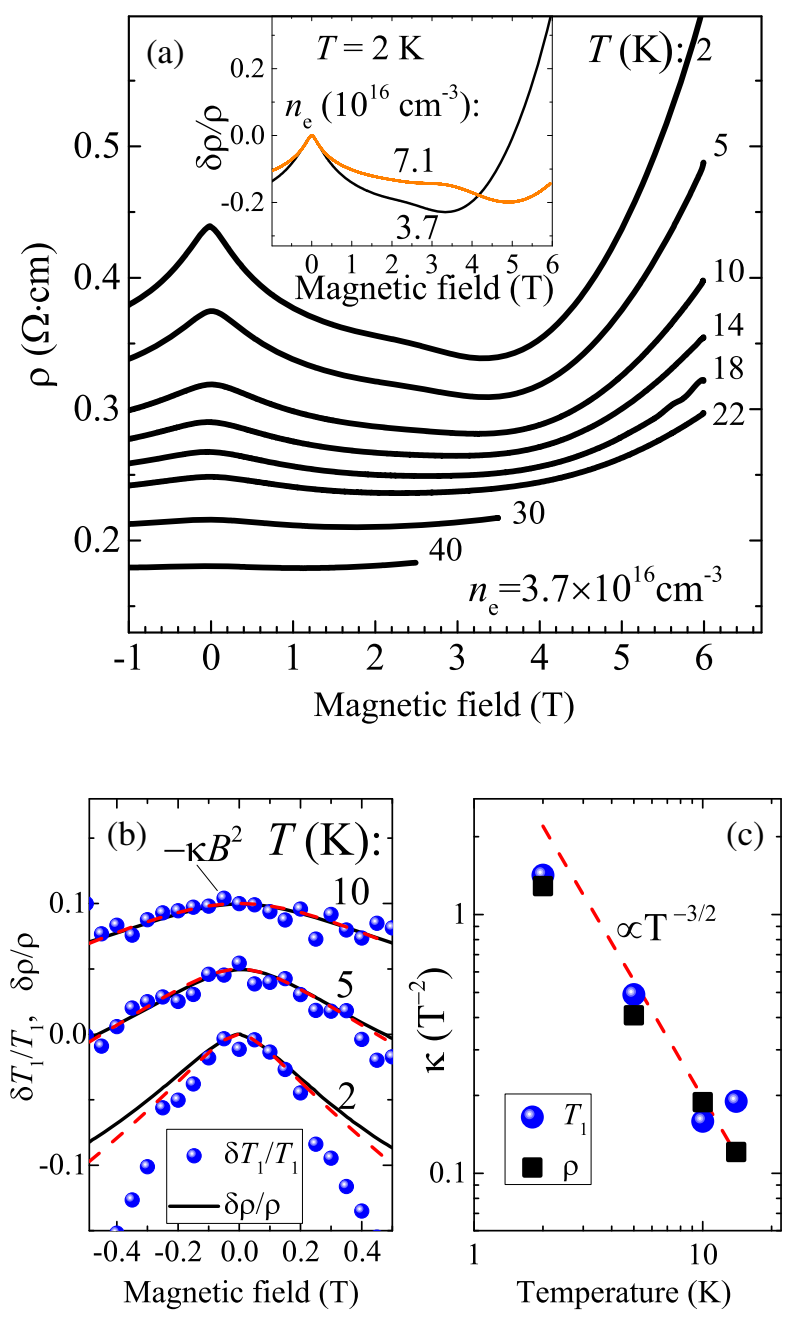

FIG. 3. Evidence of weak localization in resistivity measurements. (a) Magnetic field dependence of the resistivity $\rho$ at different temperatures. The inset shows relative variation of $\rho$ with a magnetic field for metallic samples with different electron concentrations. (b) Relative variation of $T_{1}$ (the symbols) and $\rho$ (the solid lines) with a magnetic field at different temperatures. The red dashed lines show fits to both $\delta T_{1} / T_{1}$ and $\delta \rho / \rho$ with Eq. (8). The curves are vertically shifted for clarity. (c) Curvatures of the magnetic field dependencies of $\delta T_{1} / T_{1}$ (the spheres) and $\delta \rho / \rho$ (the squares), $\kappa$ in Eq. (9), as a function of temperature. The red dashed line shows a $T^{-3 / 2}$ dependence. (a)-(c) $n_{\mathrm{e}}=3.7 \times 10^{16} \mathrm{~cm}^{-3}$.

localization effect [29-35,46]. At high fields, positive magnetoresistance is observed, presumably due to the field-induced compression of electron wave functions on donors and also possibly due to the onset of Shubnikov-de Haas oscillations. The observed behavior is qualitatively similar to that for $T_{1}(B)$ [Fig. 2(a)] and, in particular, the scale of magnetic field, destroying the weak localization, is the same. Further, the negative magnetoresistance persists in the same range of temperatures as the decrease of $T_{1}$ with $B$.

Furthermore, according to Eq. (8), the relative change of $T_{1}$ and $\rho$ due to the weak localization should be the same. 
Figure 3(b) shows these relative variations of $T_{1}$ (the spheres) and $\rho$ (the solid lines), $\delta T_{1} / T_{1} \equiv T_{1}(B) / T_{1}(0)-1$ and $\delta \rho / \rho \equiv \rho(B) / \rho(0)-1$, with magnetic field, respectively. Equation (8) is, strictly speaking, valid if the quantum corrections are small, i.e., for $\left|\delta T_{1}\right| / T_{1},|\delta \rho| / \rho \ll 1$, which is not the case in our sample with $n_{e}$ right above the MIT. Nevertheless, the measured magnetic field dependencies of $\delta T_{1} / T_{1}$ and $\delta \rho / \rho$ are in remarkable agreement in weak magnetic fields. The analysis of the asymptotic form of Eq. (8) shows that, in weak fields $B \ll B_{\phi}$,

$$
\frac{\delta T_{1}(B)}{T_{1}(0)}=\frac{\delta \rho(B)}{\rho(0)} \approx-\kappa B^{2},
$$

with the prefactor $\kappa \approx 0.048(e / m c)^{2} \sqrt{\tau_{p} \tau_{\phi}^{3}}$. In the studied temperature range, $\tau_{p}$ is constant, as found above, and $\tau_{\phi}=A / T$, where $A$ is a constant, in accordance with Refs. [35,47]. Thus, $\kappa \propto T^{-3 / 2}$. The values of curvature $\kappa$ corresponding to $T_{1}$ and $\rho$ extracted from the fit are shown in Fig. 3(c). They are in very good agreement and follow a $T^{-3 / 2}$ dependence as shown by the red dashed line.

The dashed lines in Fig. 3(b) show fits to the experiment by Eq. (8) using a reasonable set of parameters, namely, $\tau_{p}=55 \mathrm{fs}$ (temperature independent) and $\tau_{\phi}=A / T$, with $A=19 \mathrm{ps} \cdot \mathrm{K}$. Such inverse temperature dependence of the phase relaxation time was observed for a similar GaAs system [35], with $n_{e}=2.9 \times 10^{16} \mathrm{~cm}^{-3}$ giving a similar value of $A \approx 12 \mathrm{ps} \cdot \mathrm{K}$. In order to compare the value $\tau_{p}=$ $55 \mathrm{fs}$ with the previously obtained $\tau_{3}=40 \mathrm{fs}$, we calculated the ratio of $\tau_{p} / \tau_{3}$ by angular integration of the cross section of partial scattering at the screened Coulomb potential of charged impurities (see Supplemental Material [48]). For the parameters of our sample, the ratio $\tau_{p} / \tau_{3}=1.7$ and it does not reach the asymptotic value of 6 , obtained for an extremely small scattering angle [4]. Thus, the time $\tau_{3}$ obtained by considering classical DyakonovPerel relaxation is in good agreement with the time $\tau_{p}$ derived from the weak localization anomaly.

We have also studied the magnetic field dependencies of $T_{1}$ and $\rho$ for a sample with higher electron concentration $n_{e}=7.1 \times 10^{16} \mathrm{~cm}^{-3}$. The corresponding results are presented in the insets in Figs. 2(a) and 3(a) and in more detail in the Supplemental Material [48]. One can see that the effect of weak localization is reduced by a factor of about two for $n_{e}=7.1 \times 10^{16} \mathrm{~cm}^{-3}$ compared to the sample with $n_{e}=3.7 \times 10^{16} \mathrm{~cm}^{-3}$, as expected from Eq. (8), which contains $n_{e}$ in the denominator. The times $\tau_{3}, \tau_{p}$, and $\tau_{\phi}$ are similar for both samples.

In conclusion, we have demonstrated that the weak localization of electrons has a pronounced impact on their spin dynamics. The longitudinal spin relaxation time $T_{1}$ in $n$-doped GaAs, being in the metallic phase, demonstrates an anomalous decrease with increasing magnetic field at low temperatures. This decrease is due to the field-induced destruction of phase coherence for electrons resulting in the suppression of the weak localization. This shows that physics studied in transport experiments capturing the entirety of physical phenomena between the electrical contacts may be studied locally using focused optical probes of the spin dynamics. The potential of this approach will be very prominent also in two-dimensional systems where one can expect visualization of the weak-localization-induced nonexponential tails in spin polarization.

\section{ACKNOWLEDGMENTS}

We are grateful to $\mathrm{S}$. A. Crooker for providing the samples, and to E. Evers and A. Greilich for valuable advice and useful discussions. We acknowledge the financial support of the Deutsche Forschungsgemeinschaft in the frame of the ICRC TRR 160 (project A1). M. M. G. was partially supported by the RFBR Projects No. 15-52-12012 and No. 16-02-00375, Russian Federation President Grant No. MD-1555.2017.2, and the program of RAS "Nanostructures." K. V. K. and M. M. G. acknowledge support from Saint-Petersburg State University via a research grant, Grant No. 11.34.2.2012. Magnetotransport measurements were performed using research equipment of the LPI Shared Facility Center and supported by Ministry of Education and Science of the Russian Federation (Grant No. RFMEFI61717X0001). A. Yu. K. was supported by Basic research program of HSE.

[1] R. I. Dzhioev, K. V. Kavokin, V. L. Korenev, M. V. Lazarev, B. Ya. Meltser, M. N. Stepanova, B. P. Zakharchenya, D. Gammon, and D. S. Katzer, Low-Temperature Spin Relaxation in n-Type GaAs, Phys. Rev. B 66, 245204 (2002).

[2] V. V. Belykh, K. V. Kavokin, D. R. Yakovlev, and M. Bayer, Electron Charge and Spin Delocalization Revealed in the Optically Probed Longitudinal and Transverse Spin Dynamics in n-GaAs, Phys. Rev. B 96, 241201 (2017).

[3] J. G. Lonnemann, E. P. Rugeramigabo, M. Oestreich, and J. Hübner, Closing the Gap Between Spatial and Spin Dynamics of Electrons at the Metal-to-Insulator Transition, Phys. Rev. B 96, 045201 (2017).

[4] M. I. D'yakonov and V. I. Perel', Spin Relaxation of Conduction Electrons in Noncentrosymmetric Semiconductors, Fiz. Tverd. Tela 13, 3581 (1972); [Sov. Phys. Solid State 13, 3023 (1972)].

[5] K. V. Kavokin, Spin Relaxation of Localized Electrons in nType Semiconductors, Semicond. Sci. Technol. 23, 114009 (2008).

[6] M. Dyakonov and V. Kachorovskii, Spin Relaxation of TwoDimensional Electrons in Noncentrosymmetric Semiconductors, Sov. Phys. Semicond. 20, 110 (1986).

[7] Spin Physics in Semiconductors, Springer Series in SolidState Sciences Vol. 157, edited by M. I. Dyakonov (Springer International Publishing, Cham, 2017). 
[8] V. Sih, W. H. Lau, R. C. Myers, A. C. Gossard, M. E. Flatté, and D. D. Awschalom, Control of Electron-Spin Coherence using Landau Level Quantization in a Two-Dimensional Electron Gas, Phys. Rev. B 70, 161313 (2004).

[9] D. Fukuoka, T. Yamazaki, N. Tanaka, K. Oto, K. Muro, Y. Hirayama, N. Kumada, and H. Yamaguchi, Spin Dynamics of Two-Dimensional Electrons in a Quantum Hall System Probed by Time-Resolved Kerr Rotation Spectroscopy, Phys. Rev. B 78, 041304 (2008).

[10] A. V. Larionov, L. V. Kulik, S. Dickmann, and I. V. Kukushkin, Goldstone Mode Stochastization in a Quantum Hall Ferromagnet, Phys. Rev. B 92, 165417 (2015).

[11] A. V. Larionov, E. Stepanets-Khussein, and L. V. Kulik, Spin Dephasing of a Two-Dimensional Electron Gas in a GaAs Quantum Well near Odd Filling Factors, JETP Lett. 105, 238 (2017).

[12] B. I. Shklovskii, Dyakonov-Perel Spin Relaxation near the Metal-Insulator Transition and in Hopping Transport, Phys. Rev. B 73, 193201 (2006).

[13] I. S. Lyubinskiy and V. Yu. Kachorovskii, Slowing Down of Spin Relaxation in Two-Dimensional Systems by Quantum Interference Effects, Phys. Rev. B 70, 205335 (2004).

[14] G. E. Pikus and A. N. Titkov, Spin Relaxation under Optical Orientation in Semiconductors, in Optical Orientation, edited by F. Meier and B. P. Zakharchenya (North-Holland, Amsterdam, 1984).

[15] J. J. Baumberg, D. D. Awschalom, N. Samarth, H. Luo, and J. K. Furdyna, Spin Beats and Dynamical Magnetization in Quantum Structures, Phys. Rev. Lett. 72, 717 (1994).

[16] N. I. Zheludev, M. A. Brummell, R. T. Harley, A. Malinowski, S. V. Popov, D. E. Ashenford, and B. Lunn, Giant Specular Inverse Faraday Effect in $\mathrm{Cd}_{0.6} \mathrm{Mn}_{0.4} \mathrm{Te}$, Solid State Commun. 89, 823 (1994).

[17] J. S. Colton, T. A. Kennedy, A. S. Bracker, and D. Gammon, Microsecond Spin-Flip Times in n-GaAs Measured by Time-Resolved Polarization of Photoluminescence, Phys. Rev. B 69, 121307 (2004).

[18] J. S. Colton, M. E. Heeb, P. Schroeder, A. Stokes, L. R. Wienkes, and A.S. Bracker, Anomalous Magnetic Field Dependence of the $T_{1}$ Spin Lifetime in a Lightly Doped GaAs Sample, Phys. Rev. B 75, 205201 (2007).

[19] K.-M. C. Fu, W. Yeo, S. Clark, C. Santori, C. Stanley, M. C. Holland, and Y. Yamamoto, Millisecond Spin-Flip Times of Donor-Bound Electrons in GaAs, Phys. Rev. B 74, 121304 (2006).

[20] X. Linpeng, T. Karin, M. V. Durnev, R. Barbour, M. M. Glazov, E. Y. Sherman, S. P. Watkins, S. Seto, and K.-M. C. $\mathrm{Fu}$, Longitudinal Spin Relaxation of Donor-Bound Electrons in Direct Band-Gap Semiconductors, Phys. Rev. B 94, 125401 (2016).

[21] J. M. Kikkawa and D. D. Awschalom, Resonant Spin Amplification in n-Type GaAs, Phys. Rev. Lett. 80, 4313 (1998).

[22] I. A. Yugova, M. M. Glazov, D. R. Yakovlev, A. A. Sokolova, and M. Bayer, Coherent Spin Dynamics of Electrons and Holes in Semiconductor Quantum Wells and Quantum Dots under Periodical Optical Excitation: Resonant Spin Amplification Versus Spin Mode Locking, Phys. Rev. B 85, 125304 (2012).
[23] M. Oestreich, M. Römer, R. J. Haug, and D. Hägele, Spin Noise Spectroscopy in GaAs, Phys. Rev. Lett. 95, 216603 (2005).

[24] S. A. Crooker, L. Cheng, and D. L. Smith, Spin Noise of Conduction Electrons in n-Type Bulk GaAs, Phys. Rev. B 79, 035208 (2009).

[25] M. Römer, H. Bernien, G. Müller, D. Schuh, J. Hübner, and M. Oestreich, Electron-Spin Relaxation in Bulk GaAs for Doping Densities Close to the Metal-to-Insulator Transition, Phys. Rev. B 81, 075216 (2010).

[26] F. Heisterkamp, E. A. Zhukov, A. Greilich, D. R. Yakovlev, V. L. Korenev, A. Pawlis, and M. Bayer, Longitudinal and Transverse Spin Dynamics of Donor-Bound Electrons in Fluorine-Doped ZnSe: Spin Inertia versus Hanle Effect, Phys. Rev. B 91, 235432 (2015).

[27] V. V. Belykh, E. Evers, D. R. Yakovlev, F. Fobbe, A. Greilich, and M. Bayer, Extended Pump-Probe Faraday Rotation Spectroscopy of the Submicrosecond Electron Spin Dynamics in n-Type GaAs, Phys. Rev. B 94, 241202 (2016).

[28] B. L. Altshuler and A. G. Aronov, Electron-Electron Interactions in Disordered Systems, edited by A. L. Efros and M. Pollak (Elsevier, Amsterdam, 1985).

[29] H. Fritzsche and K. Lark-Horovitz, Electrical Properties of p-Type Indium Antimonide at Low Temperatures, Phys. Rev. 99, 400 (1955).

[30] J. F. Woods and C. Y. Chen, Negative Magnetoresistance in Impurity Conduction, Phys. Rev. 135, A1462 (1964).

[31] L. Halbo and R. J. Sladek, Magnetoresistance of Undoped n-Type Gallium Arsenide at Low Temperatures, Phys. Rev. 173, 794 (1968).

[32] M. Benzaquen, D. Walsh, and K. Mazuruk, Low-Field Magnetoresistance of n-Type GaAs in the Variable-Range Hopping Regime, Phys. Rev. B 38, 10933 (1988).

[33] A. Kawabata, Theory of Negative Magnetoresistance in Three-Dimensional Systems, Solid State Commun. 34, 431 (1980).

[34] A. Kawabata, Theory of Negative Magnetoresistance I. Application to Heavily Doped Semiconductors, J. Phys. Soc. Jpn. 49, 628 (1980).

[35] B. Capoen, G. Biskupski, and A. Briggs, Low-Temperature Conductivity and Weak-Localization Effect in Barely Metallic GaAs, J. Phys. Condens. Matter 5, 2545 (1993).

[36] I. S. Lyubinskiy and V. Y. Kachorovskii, Hanle Effect Driven by Weak Localization, Phys. Rev. Lett. 94, 076406 (2005).

[37] E. L. Ivchenko, Spin Relaxation of Free Carriers in Semiconductors without Inversion Center in Longitudinal Magnetic Field, Fiz. Tverd. Tela 15, 1566 (1973); [Sov. Phys. Solid State 15, 1048 (1973)].

[38] M. M. Glazov and E. L. Ivchenko, Precession Spin Relaxation Mechanism Caused by Frequent Electron-Electron Collisions, JETP Lett. 75, 403 (2002).

[39] M. M. Glazov and E. L. Ivchenko, Effect of ElectronElectron Interaction on Spin Relaxation of Charge Carriers in Semiconductors, J. Exp. Theor. Phys. 99, 1279 (2004).

[40] B. Jusserand, D. Richards, G. Allan, C. Priester, and B. Etienne, Spin Orientation at Semiconductor Heterointerfaces, Phys. Rev. B 51, 4707 (1995). 
[41] D. Richards and B. Jusserand, Spin Energetics in a GaAs Quantum Well: Asymmetric Spin-Flip Raman Scattering, Phys. Rev. B 59, R2506 (1999).

[42] V. A. Marushchak, M. N. Stepanova, and A. N. Titkov, Suppression by a Longitudinal Magnetic Field of Spin Relaxation of Conduction Electrons in Semiconductor Crystals Lacking an Inversion Center, JETP Lett. 37, 400 (1983).

[43] F. X. Bronold, I. Martin, A. Saxena, and D. L. Smith, Magnetic-Field Dependence of Electron Spin Relaxation in n-Type Semiconductors, Phys. Rev. B 66, 233206 (2002).

[44] L. P. Gor'kov, A. I. Larkin, and D. E. Khmel'nitskii, Particle Conductivity in a Two-Dimensional Random Potential, JETP Lett. 30, 228 (1979).
[45] A. P. Dmitriev, V. Y. Kachorovskii, and I. V. Gornyi, Nonbackscattering Contribution to Weak Localization, Phys. Rev. B 56, 9910 (1997).

[46] O. V. Emel'yanenko, T. S. Lagunova, and T. A. Polyanskaya, Mechanism for Relaxation of the Phase of the Electron Wave Function in n-Type GaAs, JETP Lett. 36, 246 (1982).

[47] Y. Isawa, Inelastic Scattering Time in Disordered Metals, J. Phys. Soc. Jpn. 53, 2865 (1984).

[48] See Supplemental Material at http://link.aps.org/ supplemental/10.1103/PhysRevX.8.031021 for more details on the calculation of the relation between $\tau_{p}$ and $\tau_{3}$ and on the experimental results related to the high-density sample. 